\title{
marges Marges
}

revue d'art contemporain Revue d'art contemporain

26 | 2018

Instrumentalisations de l'art

\section{Un rendez-vous raté : communistes et surréalistes dans les années 1930}

A Missed Appointment: Communists and Surrealists in the 1930s

\section{Gwenn Riou}

\section{(2) OpenEdition}

1 Journals

Édition électronique

URL : http://journals.openedition.org/marges/1360

DOI : 10.4000/marges.1360

ISSN : 2416-8742

Éditeur

Presses universitaires de Vincennes

\section{Édition imprimée}

Date de publication : 19 avril 2018

Pagination : 10-23

ISBN : 978-2-84292-805-6

ISSN : $1767-7114$

\section{Référence électronique}

Gwenn Riou, «Un rendez-vous raté : communistes et surréalistes dans les années 1930 », Marges [En ligne], 26 | 2018, mis en ligne le 19 avril 2020, consulté le 07 janvier 2021. URL : http://

journals.openedition.org/marges/1360 ; DOI : https://doi.org/ERREUR PDO dans /localdata/www-bin/ Core/Core/Db/Db.class.php L.34 : SQLSTATE[HY000] [2006] MySQL server has gone away 


\section{Un rendez-vous raté: communistes et surréalistes dans les années 1930}

\begin{abstract}
/1 André Breton, «Caractère de l'évolution moderne et ce qui en participe » (1922), cité par Gérard Durozoi dans Histoire du mouvement surréaliste, Paris, Hazan, 1997, p. 44.
\end{abstract}

/ 2 L'Humanité, 2 juillet 1925, p. 1.

/3 L'Humanité, 21 septembre 1925, p. 2. Le texte est également publié dans La Révolution surréaliste ${ }^{\circ} 5$, octobre 1925, p. 31-32.

/4 Voir Michel Trebitsch, "Le groupe Philosophies et les surréalistes (1924-1925) », Mélusine $\mathrm{n}^{\circ} \mathrm{XI}$, « HistoireHistoriographie ", 1990, p. 63-86; Marguerite Bonnet (sld), Archives du surréalisme, t. 2, "Vers l'action politique, juillet 1925-avril 1926 », Paris, Gallimard, 1988, p. 34, p. 75, p. 103.
Après plusieurs années de tractations et de négociations, l'Association des Écrivains et Artistes révolutionnaires (AÉAR) est créée en mars 1932. Cette organisation qui compte dans ses rangs des personnalités comme Paul Nizan, Louis Aragon, Boris Taslitzky, André Fougeron, Francis Jourdain, Édouard Pignon, Paul Signac, Henri Cartier-Bresson, etc. s'impose rapidement comme une plateforme artistique et littéraire de premier ordre en France.

L'histoire de cette organisation artistique est liée, d'une part, à l'histoire du Parti communiste français (PCF), de l'Internationale communiste (IC ou Komintern) et d'autre part à l'histoire de la littérature. En effet, lorsque l'on se penche sur les rapports qu'entretient le Parti communiste avec les acteurs du champ culturel, il apparaît que les écrivains jouent un rôle majeur dans la conception, l'élaboration et la structuration d'une création artistique en rapport avec les volontés politiques et idéologiques du Parti. Parmi ces écrivains, nous retrouvons les surréalistes.

Alors que ces derniers suivent encore au début des années 1920 le mouvement dada dirigé par Tristan Tzara, certains d'entre eux, dont André Breton, aspirent à la révolution, « une révolution quelconque, aussi sanglante qu'on voudra/1 ». Cette prise de position participe au divorce entre les dadaïstes et ceux qui deviendront les surréalistes. Ceux-ci signent en 1925 l'«Appel aux travailleurs intellectuels/2 » dans l'Humanité et se positionnent contre la guerre du Rif et pour le droit des peuples « à disposer d'eux-mêmes ». Deux mois plus tard, ils publient dans l'organe central du PCF, «La Révolution d'abord 
et toujours/3 " et s'engagent, de ce fait, de manière durable, sur le terrain de la politique en reprenant les principaux thèmes défendus par les communistes: antimilitarisme, anticolonialisme, anti-impérialisme, etc. Ce texte rappelle qu'à cette période les surréalistes, en collaboration avec d'autres intellectuels, évoquent le souhait de mettre au point une organisation culturelle révolutionnaire affiliée à l'IC/4. Quant au PCF, celui-ci ne commence vraiment à s'intéresser à la question de la création artistique et notamment littéraire qu'à la fin des années 1920. Jusqu'alors les intellectuels n'intéressent pas les dirigeants du Parti; plus encore, ils s'en méfient. Le PCF suit en effet une tendance ouvriériste qui l'amène à prendre l'aspect d'une secte persécutée dont la combativité prolétarienne constitue son essence/5.

Ce texte n'a pas pour but de revenir sur l'histoire des relations qu'entretiennent surréalistes et communistes dans les années 19201930 mais plutôt de s’interroger sur un épisode méconnu de leur histoire commune: la constitution de l'AÉAR. Il sera donc question de s'attarder sur la volonté du Komintern, du PCF et des surréalistes de créer une organisation artistique révolutionnaire en France. Ainsi, l'idée que l'AÉAR est une création ex nihilo de l'IC sera remise en question/6. L'objet de ce texte n'est pas tant de savoir à qui revient la paternité de l'Association que de comprendre comment le Parti communiste a réussi à y asseoir son hégémonie. Celui-ci est, au début des années 1930, en pleine réflexion sur la stratégie politique qu’il doit adopter. La ligne sectaire dite "classe contre classe » que le Parti suit depuis 1928 ne porte pas ses fruits et son influence « atteint son étiage historique/7 "; de plus, malgré les recommandations de I'IC, il n'a toujours pas créé d'organisation d'artistes et d'écrivains révolutionnaires/8. Au regard de ce contexte nous ne pouvons que nous interroger: les communistes ont-ils manipulé, voire instrumentalisé, les surréalistes dans le but de répondre aux exigences du Komintern? La mise en lumière des diverses tractations qui ont eu lieu entre surréalistes et communistes pendant les années 1930-1932 permettront d'esquisser un élément de réponse à cette question.

\section{Les surréalistes et le Parti communiste}

De la même façon que les communistes sont dépositaires d'un projet révolutionnaire totalisant, les surréalistes aspirent à transformer l'homme et la société, ils se revendiquent ainsi tous les deux à l'avantgarde du progrès. La différence entre ces deux groupes est pourtant de taille: l'un est un parti politique structuré à l'échelle nationale et mondiale; l'autre, s'il est également organisé, ne rassemble, sur les bases de l'action poétique et artistique, que peu de personnes.
/5 Voir Jean Touchard, "Le Parti Communiste Français et les Intellectuels (1920-1939) », Revue française de science politique $\mathrm{n}^{\circ} 3,1967$, p. 470; Henri Béhar (sld), Le Surréalisme dans la presse de gauche (1924-1939), Paris, Paris-Méditerranée, 2002, p. 8-9.

/6 Les ouvrages ou articles qui traitent de l'Association abordent peu les conditions de sa création car il n'existe pas, à ce jour, d'archives de l'organisation. II est donc admis qu'elle résulte uniquement des volontés du Komintern, ce que cet article tend à nuancer. Sur l'AÉAR, voir en particulier Nicole Racine, «L'Association des Écrivains et Artistes révolutionnaires (AÉAR). La revue Commune et la lutte idéologique contre le fascisme (1932-1936) ", Le Mouvement social $\mathrm{n}^{\circ} 54$, janvier-mars 1966 , p. 29-47 ; Jean-Pierre Morel, Le Roman insupportable. L'Internationale littéraire et la France (1920-1932), Paris, Gallimard, 1985.

I7 Stéphane Courtois et Marc Lazar, Histoire du Parti communiste français, Paris, PUF,

1995, p. 109. À propos de «l'étiage » du PCF et de ses causes à la fin des années 1920 et au début des années 1930, voir, Yves Santamaria, Histoire du Parti communiste français, Paris, La Découverte, 1999, p. 27-29.

/8 Voir Jean-Pierre Morel, op. cit. 
/9 Georges Sadoul, lettre à André Thirion, 10 octobre 1926 , cité par Valérie Vignaux, «Georges Sadoul et le surréalisme (1925-1932) ou chronique d'un itinéraire intellectuel ", Les Annales de la Société des amis de Louis Aragon et Elsa Triolet $\mathrm{n}^{\circ} 17$, 2016, p. 19.

/10 En février 1927, André Breton, Louis Aragon, Paul Éluard, Benjamin Péret et Pierre Unik adhèrent au PCF. André Thirion, lui est militant communiste depuis 1925 .

/11 Georges Sadoul, lettre à André Thirion, 25 février 1927 , cité par Valérie Vignaux, op. cit., p. 20.

/12 ibid.

/13 André Breton, "Légitime défense ", La Révolution surréaliste $n^{\circ} 8$, décembre 1926 , p. 31.

/14 ibid., p. 32.
En octobre 1926, Sadoul écrit à Thirion que « Breton veut [ce rapprochement avec le Parti communiste], sous réserve qu'on leur laissera leur liberté d'action en dehors du Parti, et je crois que Breton le voulant, les autres le voudront et qu'il n'y aura pas de crise du fait de cela/9 ». Les surréalistes pensent donc, à ce moment-là, être capables de concilier une allégeance consentie (terme qui ne paraît pas exagéré dans cette période dite de « bolchévisation » du PCF) et l'autonomie de leurs activités créatrices. Les surréalistes espèrent alors profiter de l'implantation du parti communiste et de leurs relais de diffusion pour faire entendre leur voix qui est relativement peu audible au-delà des cercles d'avant-garde et ainsi s'imposer comme un groupe artistique révolutionnaire de premier ordre.

Si l'adhésion au début de l'année 1927 des surréalistes/10 au Parti est un évènement essentiel dans l'histoire du groupe, du côté du PCF cela ne s'apparente guère plus qu'à un détail. Le peu de considération des surréalistes de la part des communistes se comprend d'autant plus qu'en matière de culture et plus précisément de littérature, c'est à Henri Barbusse qu'incombe, depuis les années 1925-1926, le rôle d'organiser en France une réunion d'écrivains et d'artistes révolutionnaires. Cependant, malgré les insistances des dirigeants du Komintern qui ont chargé Barbusse de cette tâche, celui-ci n’y répond pas franchement.

\section{L’adhésion des surréalistes et après?}

Quelques jours à peine après son adhésion, « [Breton] veut plaquer le Parti/11 ». Il est en effet demandé à l'écrivain de placer sa revue, La Révolution surréaliste, sous le contrôle du Comité central du PCF, ce qu'il refuse. De surcroît, il est attaqué par un membre du Parti qui l'accuse d'adhérer au PCF dans le seul but de prendre d'assaut l'Humanité/12. Le quotidien communiste a en effet fait l'objet de violentes attaques de la part de Breton. En décembre 1926, dans La Révolution surréaliste, il qualifie le journal de «puéril, déclamatoire, inutilement crétinisant » mais aussi, d'« illisible [et] tout à fait indigne du rôle d'éducation prolétarienne qu'il prétend assumer ». Surtout, le surréaliste trouve que «les courants véritables de la pensée moderne s'y manifestent moins que partout ailleurs. La vie des idées y est à peu près nulle/13 ». Ce reproche s'adresse plus précisément à Barbusse, alors directeur littéraire du journal dont Breton écrit qu'« intellectuellement parlant, il n'est pas non plus, à l'exemple des écrivains que nous, surréalistes, faisons profession d'admirer, un éclaireur. M. Barbusse est, sinon un réactionnaire, du moins un retardataire, ce qui ne vaut peut-être pas mieux/14 ». 
Cette dernière attaque est de taille car pour les surréalistes la notion de progrès, en littérature ou plus généralement en matière de création artistique, va de pair avec une avancée sociale et politique/15. Barbusse est donc l'opposé de ce à quoi ils aspirent. Plus encore, l'auteur du Feu aurait fait l'éloge de Paul Claudel et de Jean Cocteau, des "auteurs de poèmes patriotiques infâmes, de professions de foi catholique nauséabondes, profiteurs ignominieux du régime et contre-révolutionnaires fieffés/16 ». L'éloge de ces écrivains «bourgeois " par un auteur communiste à cette période dure de l'histoire du Parti est tout simplement inconcevable pour les surréalistes. En publiant ce texte, Breton opère une double manœuvre. Premièrement, il démontre que le surréalisme est le principe de création artistique le plus à même d'incarner et de participer au développement révolutionnaire; deuxièmement, en attaquant le point de vue de Barbusse sur Cocteau et Claudel, il s'impose sur le terrain de la politique du Parti et de sa ligne dite "bolchévique » qui entraîne avec elle son lot de sectarisme.

Jusqu'en 1930, la relation entre les communistes et les surréalistes est fluctuante, leur engagement pour la cause révolutionnaire est loué mais leur intérêt pour le freudisme est critiqué. Peu actifs au sein du Parti, ils laissent « les questions politiques aux militants pour se consacrer au domaine littéraire qui relève de leur compétence/17 ", comme le souligne Carole Reynaud-Paligot. II semble alors qu'ils soient dans l'attente du moment propice où ils pourront s'imposer comme le groupe dépositaire de la politique artistique et littéraire du PCF.

La situation semble en effet leur donner raison. D'une part, malgré les insistances de l'Internationale communiste, Barbusse ne fait toujours rien pour créer un regroupement d'artistes et d'écrivains révolutionnaires. Il lance cependant la revue Monde en juin 1928 mais son ouverture à un ensemble d'intellectuels anticapitalistes (et non seulement communistes) vaut à la publication de se faire reprocher son éclectisme par Moscou. Monde n'est pas considérée comme assez révolutionnaire et la méfiance des appareils communistes envers son fondateur va s'accentuer pour atteindre son paroxysme en 1930. D'autre part, en septembre 1929, des militants communistes réalisent un véritable coup d'état au sein de l'Humanité. Avec l'appui officiel du Parti, ils occupent les locaux du quotidien et expulsent six rédacteurs/18. Il est reproché au journal de ne pas défendre pleinement la ligne politique du Parti et de jouer le jeu de la bourgeoisie. Pour Jean-Pierre Morel, ce « putsch » est révélateur de la volonté du Parti d'encadrer plus fermement les écrivains et la "presse sympathisante» mais aussi d'évincer les «modes de regroupement et d'influence moins politiques/19 ».
/15 Sur l'idée d'un lien entre progressisme politique et artistique chez les surréalistes et le Parti communiste voir Guillaume Bridet, "Tensions entre les avantgardes: le surréalisme et le Parti communiste », Itinéraires $n^{\circ} 4,2001$, [http://itineraires.revues. org/1366; DOI : 10.4000/ itineraires.1366], consulté le 12 février 2015.

/16 ibid., p. 33.

/17 Carole Reynaud-Paligot, Parcours politique des surréalistes, 1919-1969, Paris, CNRS, 2010, p. 107.

/18 À ce propos, voir Bureau politique du Parti communiste français, « Une déclaration du Bureau politique », l'Humanité, 3 septembre 1929, p. 1 et Henri Barbé, "La signification de notre "coup d'état" ", l'Humanité, 9 septembre 1929, p. 1.

/19 Jean-Pierre Morel, op. cit., p. 244. 
/20 André Breton, "Second manifeste du surréalisme ", La Révolution surréaliste $\mathrm{n}^{\circ} 12$, décembre 1929 , p. 1-17.

/21 ibid., p. 33.

/22 ibid.

/23 André Thirion, Révolutionnaires sans révolution, Paris, Le Pré aux clercs, 1988 , p. 269-270.

/24 Leur refus de la littérature prolétarienne et la part belle qu'ils font à la psychanalyse ne semblent pas, à ce moment-là, déranger outre mesure les instances communistes, leur critique de Monde et d'Henri Barbusse semblant primer sur le reste.

/ 25 Bureau International de la Littérature Révolutionnaire, "Question », Le Surréalisme au service de la révolution $n^{\circ} 1$, juillet 1930, p. 1.
Il semble qu'en cette fin d'année 1929 toutes les conditions soient réunies pour que les surréalistes s'imposent auprès des instances communistes nationales et internationales. Ce n'est donc pas un hasard s'ils décident de publier dans le dernier numéro de $L a$ Révolution surréaliste non seulement le "Second manifeste du surréalisme/20", mais également l'article d'Aragon, "Monde, 23 novembre/21", qui constitue une violente charge contre Barbusse et son journal qualifié d'« ordure confusionnelle/22 ». Quant au "Second manifeste », rappelons rapidement que s'il est connu pour contenir de nombreuses prises de positions en faveur du freudisme et des références à Trotski, il se situe néanmoins dans la droite ligne politique du « classe contre classe » prôné par le Parti. Thirion écrit à ce propos que "le "Second Manifeste" avait donné l'aval à cette ligne qui constituait la trame des numéros 1 et 2 du Surréalisme au service de la Révolution [...]. Un de nos objectifs essentiels était de dissiper le brouillard de confusion qui entourait l'hebdomadaire Monde, de situer celui-ci parmi les adversaires les plus dangereux de la classe ouvrière [...]. Notre pensée se soumettrait aux règles du matérialisme historique et du léninisme, mais chacun reconnaissait que les surréalistes devaient encore accomplir des progrès dans l'interprétation correcte et l'usage des saintes écritures/23 ».

Notons également le changement de titre de la publication qui renforce l'idée d'allégeance du surréalisme au communisme. Ainsi, entre la publication du dernier numéro de la Révolution surréaliste et le premier du Surréalisme au service de la Révolution, le décor est planté: critique des non-communistes; connaissance des « saintes écritures »; forme d'autocritique (qui doit amener les surréalistes à continuer leur travail idéologique).

Tous les éléments semblent réunis pour que les surréalistes puissent rentrer dans les grâces de l'appareil communiste/24. Il ne leur reste qu'à déclarer officiellement leur allégeance. Cela est rendu possible lorsqu'au printemps 1930 Breton reçoit le télégramme suivant: "Bureau International Littérature Révolutionnaire [BILR] prie répondre question suivante laquelle sera votre position si impérialisme déclare guerre aux Soviets/25? ». La réponse rédigée par Aragon et Breton est sans équivoque: "Camarades si impérialisme déclare guerre aux Soviets, notre position sera conformément aux directives Troisième Internationale position membres du Parti communiste français. Si estimiez en pareil cas un meilleur emploi possible de nos facultés sommes à votre disposition pour mission précise exigeant tout autre usage de nous en tant qu'intellectuels stop vous soumettre suggestions serait vraiment présumer de notre rôle 
et des circonstances. Dans situation actuelle de conflit non armé croyons inutile attendre pour mettre au service de la révolution les moyens qui sont plus particulièrement les nôtres/26. ». Envoyée directement à Moscou, cette réponse ne reste pas confidentielle. Elle est publiée en France dans le premier numéro du Surréalisme au service de la révolution, puis en octobre en URSS dans Le Courrier de la Littérature étrangère et dans La Gazette littéraire, au moment même où Aragon est à Moscou.

\section{L'Association des Artistes et Écrivains Révolutionnaires (AAÉR)}

Aragon est dans la capitale soviétique depuis le début du mois d'octobre 1930 lorsqu'il est rejoint par Sadoul. Ce qui devait être à l'origine un périple informel se transforme en un voyage officiel. Les deux Français sont en effet invités à participer à la « Deuxième conférence internationale des écrivains révolutionnaires " (appelée plus communément Congrès de Kharkov), organisée par le BILR qui dépend de l'IC. Le but de ce congrès est de faire le point sur la production littéraire (et dans une moindre mesure artistique) communiste à travers le monde et de créer, sous l'égide du Komintern, une union internationale littéraire - qui prend, après le congrès, le nom d'Union Internationale des Écrivains révolutionnaires (UIÉR). Les surréalistes se préparent alors, comme le souligne Aragon, à travailler «avec les membres dirigeants du Bureau en question qui disent à l'heure actuelle préférer travailler avec des gens qui ont une plateforme littéraire différente de la leur, mais qui ont la même plateforme politique qu'eux (c'est-à-dire en France les surréalistes) plutôt qu'avec des littérateurs qui semblent plus voisins d'eux littérairement mais qui politiquement sont des bourgeois (Barbusse et autres)/27 ».

On comprend alors qu'Aragon et Sadoul veulent s'imposer devant les plus hautes instances de l'Internationale comme étant les seuls à pouvoir faire respecter les mots d'ordre du BILR en France. Pour cela ils se doivent de créer une organisation affiliée à ce dernier qui leur permettra d'assurer de manière pérenne leur mainmise sur la création littéraire et artistique révolutionnaire dans l'Hexagone. Aragon poursuit sa lettre: "Nous comptons, Georges et moi, sur votre confiance à tous, sur la tienne pour parler en notre nom à Kharkov où il nous sera peut-être donné de liquider, de contribuer à liquider Monde et tout ce qui s'ensuit. [...] Nous écrivons un petit article en collaboration pour la [Gazette littéraire] [...] pour y définir notre point de vue sur la situation littéraire en France, la possibilité d'organisation analogue au Bureau en France/28. ». Quelques jours
/26 Anonyme [Louis Aragon et André Breton], "Réponse », ibid.

/ 27 Lettre à André Breton, 22 octobre 1930, dans Louis Aragon, Lionel Follet (sld), Lettres à André Breton: 1918-1931, Paris, Gallimard, 2011, p. 390.

/ 28 Lettre à André Breton, 22 octobre 1930, ibid., p. 392. 
/29 Louis Aragon et Georges Sadoul, copie en français de la «Déclaration des surréalistes français ", ibid., p. 412.

/30 Artiste peintre né à Vienne en 1902 et mort à Nice en 1959.

/31 André Thirion, op. cit., p. 273.

/32 Victor Bauer cité dans ibid.

/33 André Thirion, André Breton, "Statuts provisoire de l'AAÉR », 28 novembre 1930 , cote Ms 3485, Nantes, Bibliothèque municipale de Nantes, [http:// www.andrebreton.fr/ work/5660010 009416o], consulté le 29 décembre 2016.

/34 ibid. après l'envoi de cette lettre, la Gazette littéraire publie la « Déclaration des surréalistes français/29", dans laquelle ces derniers annoncent publiquement leur volonté de fonder en France une section du Bureau international de la littérature révolutionnaire.

Au même moment, en France, Breton et Thirion élaborent ce projet d'organisation avec l'aide d'un certain Victor Bauer/3o. Ce dernier, qui se déclare membre de l'appareil clandestin du Parti "surgit " dans le groupe surréaliste à l'automne 1930 et propose d'emblée le « rassemblement de tous les artistes révolutionnaires dans une grande association, prétendant que seuls les surréalistes [sont] capables de mener cette tâche à bien/31 ॥. Il soumet alors à Breton un texte résumant ses intentions quant à la constitution d'un tel groupement. Ce texte, selon Thirion, présente la caractéristique de faire le lien entre le freudisme et le marxisme et s'inscrit de fait dans les recherches surréalistes. Bauer précise même aux surréalistes qu'il a l'aval des «camarades [communistes] étrangers/32 ». L'arrivée de ce personnage, inconnu jusque-là, sa position au sein du Parti et ses intentions à ce moment précis, soulèvent des questions. II n'en reste pas moins que son texte est accepté. Retravaillé par Breton et Thirion en octobre-novembre, il résume les « principes généraux » de l'AAÉR en cours d'élaboration.

Le texte commence d'emblée par des références à la psychanalyse et à la biologie et s'éloigne ainsi du matérialisme qui prévaut dans la plupart des textes rédigés par des communistes. Cependant, le principe de la dialectique cher aux marxistes n'est pas absent. L'artiste est alors un "produit biologique et sociologique » dont " la base matérielle de son existence repose sur les conditions économiques de la société dans laquelle il vit/33 ». Cette mise au point effectuée, les auteurs précisent leurs intentions et les buts de la future association: "S'il ne s'organise pas, l'artiste ne court donc aucune chance d'améliorer sa situation économique, situation aussi mauvaise, sinon pire, que celle du prolétariat. Le seul moyen qu'il ait de sauvegarder l'indépendance de ses recherches, c'est de lutter à côté des travailleurs exploités contre la société capitaliste. Le guide dans cette lutte ne peut être que l'organisation du prolétariat conscient: le parti communiste. Pour l'artiste, la nécessité s'impose de créer son propre syndicat, de faire ainsi valoir par le fait que le sort du prolétariat révolutionnaire est lié au sien/34. ».

Si le terme d'« indépendance » est employé ici par opposition aux contraintes du système capitaliste, on peut imaginer qu'il vaut aussi pour l'ensemble des systèmes étatiques et politiques. Insidieusement, Breton et Thirion soulèvent donc la question de l'autonomie du champ artistique vis-à-vis du politique et renvoient de fait à 
Trotsky qui considère "l'art en tant qu'art, c'est-à-dire en tant que domaine particulier, spécifique, de l'activité humaine/35 ».

Adjoints aux "principes généraux » de l'AAÉR, les "statuts provisoires » de l'Association précisent les conditions d'adhésion des membres. Ainsi sera accepté « tout peintre ou sculpteur qui pourra fournir la preuve qu'il est l'auteur d'une peinture ou d'une sculpture susceptible de servir la propagande révolutionnaire/36 ». Est alors considéré comme révolutionnaire tout ce qui est «compatible avec la morale ou la philosophie marxiste/37 » et qui peut servir à la propagande de l'IC. Cependant, ne peuvent pas être acceptés et seront exclus tous ceux qui collaborent ou collaboreront à des publications ou organismes jugés contre-révolutionnaires. On pense évidemment à toutes les émanations de la « bourgeoisie » et du capitalisme mais également à Monde et à son directeur, Barbusse.

Enfin, un comité provisoire composé de Breton, d’Éluard, de Meyer et de Thirion est constitué. Aragon et Sadoul sont également pressentis pour intégrer le comité provisoire. Cependant, précise Thirion, il faut choisir entre l'un ou l'autre afin de ne pas avoir « tant de nos amis dans ce comité/38».

\section{Le Congrès de Kharkov}

Le 9 novembre 1930, lors de sa première intervention au congrès, Aragon proclame: "Nous ne sommes pas ici comme surréalistes, mais comme communistes/39. ». C'est le contraire de ce qu'il a écrit quelques jours plus tôt à Breton. Parallèlement, Sadoul informe Thirion du déroulement du congrès et tente de le rassurer en écrivant qu'« il a fallu une fois de plus confronter le matérialisme historique et le surréalisme, montrer que celui-ci ne contredisait pas celui-là. Nous nous en sommes assez bien tiré je crois aux yeux de nos juges et notre position s'est trouvée renforcée d'autant/40. ».

Nous ne pouvons que nous interroger sur le double-jeu joué par Aragon et Sadoul et sur ce qu'il s'est réellement passé durant ce congrès. Sans doute les deux Français se sont aperçus que leur position surréaliste n'était pas viable à Kharkov. Peut-être pensentils pouvoir renier le surréalisme en URSS afin de revenir en France avec la charge d'organiser l'association pour ensuite l'orienter dans la voie qu'ils souhaitent (le surréalisme). Il est probable que le BILR se soit servi d'eux pour forcer la main à Barbusse afin que ce dernier réoriente sa revue et se décide à s'occuper de l'organisation souhaitée. II n'est pas possible d'être affirmatif d'autant qu'au même moment (aux alentours du 12 novembre) des tractations ont lieu à Paris. Thirion annonce en effet à Sadoul qu'il a déposé le
/35 Léon Trotski cité par Jean-Marie Brohm, " Les marxismes et les arts ", Prétentaine $\mathrm{n}^{\circ} 6$, 1996, p. 183.

/36 André Thirion, André Breton, «Statuts provisoire de l'AAÉR », op. cit.

/37 ibid.

/38 André Thirion, lettre à André Breton, non datée (automne 1930), cote Ms 3485, Nantes, Bibliothèque municipale de Nantes, [http:// www.andrebreton.fr/ work/5660010 0020900], consulté le 30 décembre 2016.

/39 « Séance du 9-XI 1930. Séance du matin ", Littérature de la révolution mondiale numéro spécial « Deuxième Conférence Internationale des Écrivains Révolutionnaires », 1931, p. 183.

/40 Georges Sadoul, lettre à André Thirion, 13 novembre 1930, cité par Valérie Vignaux, op. cit., p. 13. 
/41 Paul Vaillant-Couturier cité par André Thirion, lettre à Georges Sadoul, 12 novembre 1930 , cité dans ibid., p. 13-14.

/ 42 Voir la notice de Marguerite Bonnet pour Misère de la poésie, dans André Breton, Euvres complètes, t. II, présenté par Marguerite Bonnet, Paris, Gallimard, coll. « bibliothèque de la Pléiade ", 1992, p. 1291-1300.

/ 43 Louis Aragon, " Front rouge ", Littérature de la Révolution mondiale $n^{\circ}$ 1, juillet 1931, p. 39-46.

/44 Anonyme, "L'inculpation d'Aragon ", l'Humanité, 9 mars 1932, p. 2.

145 ibid.

/46 Jean Fréville, lettre à Bruno Jasienski, 20 janvier 1932, cité par Jean-Pierre Morel, op. cit., p. 459.

/47 Les recherches menées par Nicole Racine dans les archives du Komintern montrent bien à quel point les instances communistes sont suspicieuses quant à Aragon. Dans le dossier de ce dernier on peut lire ces quatre lignes écrites en 1936: «Aragon, fils d'Andrieux, ancien préfet de Police. Surréaliste, dirige la Maison de la Culture (ancienne AÉAR). Vaillant-Couturier principal responsable, Aragon Secrétaire général. Aragon en 1925 publia un article violemment
8 novembre les statuts de l'AAÉR auprès du responsable des intellectuels au sein du Parti, Paul Vaillant-Couturier et a demandé à rencontrer Maurice Thorez alors Secrétaire général du PCF. S’il ne reçoit pas de réponse de ce dernier, il rencontre néanmoins Vaillant-Couturier qui lui dit qu'il « faut [...] renier le "Second manifeste", enlever purement et simplement le mot surréalisme de toute votre activité, renier le désespoir. Remarquez que pour ma part, cela n’a aucune importance. Je considère le surréalisme comme un mouvement d'une importance révolutionnaire très grande, l'activité surréaliste comme parfaitement défendable, le désespoir comme parfaitement naturel, mais politiquement (!!!)/41 ».

Il y a donc une désapprobation du surréalisme par les communistes français. Il est probable qu'ils soupçonnent les surréalistes de vouloir garder la mainmise sur la future organisation. Pour le PCF, enlever les mentions relatives au surréalisme permettrait d'éclaircir ce point. Plus que cela, Vaillant-Couturier a sans doute l'intention de placer des communistes non surréalistes à des postes clés de l’Association. Quelques jours après le 12 novembre, le projet porté par Thirion, Breton et Éluard, est adopté lors d'une assemblée où siègent les communistes Léon Moussinac et Vaillant-Couturier. II n'est pas possible de savoir si les références au surréalisme présentes dans les « principes généraux » sont évincées de cette version adoptée, il n'empêche que l'officialisation de l'organisation semble être imminente.

\section{Le retour d'URSS, confrontation, division, création}

Lors de leur retour en France, Aragon et Sadoul avouent à Breton qu'ils ont dû renier le surréalisme et notamment le « Second manifeste » avec ce qu'il comporte comme références au freudisme et au trotskisme. Ce geste leur a permis, selon eux, de se légitimer aux yeux du BILR. Malgré le tollé que provoque chez les surréalistes le reniement des deux congressistes, l'affaire semble entendue, du moins jusqu'au début de 1932, lorsqu'éclate «L'Affaire Aragon/42 ». Le 16 janvier 1932, le poète est poursuivi en justice pour « excitation de militaires à la désobéissance » suite à la publication de son poème «Front Rouge/43 ». Les surréalistes, Breton en première ligne, défendent alors l'inculpé en publiant la pétition "L'Affaire Aragon ». Toutefois, cette pétition est dénoncée par l'Humanité qui reproche aux surréalistes non seulement d'exiger l'immunité politique "pour les poètes et pour les poètes seulement ", mais surtout de se battre essentiellement sur le front artistique au lieu de "défendre le contenu du poème/44 ». Pour l'organe du PCF, le « révolutionnarisme [des surréalistes] n'est que verbal/45 ». 
Cette attaque, et de fait la mise à l'index des surréalistes par les communistes, interroge quant au rôle que peuvent jouer leurs suspicions mutuelles et leurs conceptions esthétiques dans leur entente relative. La lettre envoyée le 20 janvier 1932 par Jean Fréville au dirigeant de l'UIÉR, Bruno Jasienski, à propos des surréalistes peut nous éclairer à ce sujet: "Dès [que les surréalistes] ont appris la création d'une Association des Écrivains révolutionnaires de France, [ils] ont envoyé leur adhésion (Breton, Éluard, Aragon, etc.). Nous leur poserons des questions précises: la première sera de rompre avec leur passé idéologique et littéraire. Je ne pense pas qu'ils acceptent. Leur revue: Le surréalisme au service de la Révolution, dont deux numéros viennent de paraître [...] dévoile encore une fois leur essence d'écrivains petitbourgeois, irrémédiablement étrangers et hostiles au prolétariat, malgré le ronflement de leurs phrases révolutionnaires. Dans un des numéros de cette revue, Aragon, pourtant membre du parti communiste, se livre à une révision véritable, et que nous ne tolérons pas, de la plateforme de Kharkov et continue à faire l'éloge de ses amis surréalistes. Avant d'accepter Aragon comme membre de l'Association des Écrivains révolutionnaires, nous lui demanderons de rompre avec le surréalisme et de condamner l'article qu'il vient d'écrire. Faute de quoi nous [...] demanderons à l'Association de prendre contre Aragon les mesures qui s'imposent/46. ॥.

Il apparaît donc que le PCF constitue une association d'écrivains affiliée à l'Internationale, et ce sans le groupe de Breton qui s'y était pourtant attelé. Cette Association des Écrivains révolutionnaires est à cette date la première version de l'AÉAR, qui sera officiellement constituée en mars. Qu'en est-il de l'Association surréaliste dont les statuts ont été déposés à l'automne 1930? Aucune information concernant l'existence officielle de cette organisation ne nous est parvenue. Il semble alors que le PCF a dépossédé les surréalistes de leur projet et se l'est accaparé. Sûrement ne leur faisaient-ils pas confiance, surtout à Aragon/47. La publication du tract d'Aragon et de Sadoul, "Aux intellectuels révolutionnaires », à leur retour d'URSS en décembre 1930, puis un an plus tard de l'article d'Aragon «Le surréalisme et le devenir révolutionnaire/48 " ne peuvent que leur donner raison/49. Ces publications qui font la part belle au freudisme et à l'idéalisme sont de trop pour le Parti : les surréalistes sont écartés de leur projet d'association.

\section{L'AÉAR sans les surréalistes?}

« L’Affaire Aragon » est alors un prétexte pour attaquer le surréalisme et pour préciser les attentes du PC en matière de création artistique antisoviétique. Renseignements communiqués par le camarade Fried. ». Cité par Nicole Racine, «Aragon dans les archives. Paris-Moscou 1930-1936 ", Faites entrer l'infini $\mathrm{n}^{\circ} 25$, juin 1998, p. 11.

/48 Louis Aragon, "Le surréalisme et le devenir révolutionnaire ", Le Surréalisme au service de la révolution $\mathrm{n}^{\circ} 3$, décembre 1931, p. 3-9.

/49 Nous savons aujourd'hui que le texte de Louis Aragon a été écrit à la demande d'André Breton et de Paul Éluard, comme en témoigne cette lettre qui lui a été envoyée en octobre 1931: « À Kharkov tu as déclaré que tu traiterais quand on te le demanderait de la position des surréalistes en France. C'est nous qui croyons le moment venu de te le demander ». André Breton et Paul Éluard, lettre à Louis Aragon, 22 octobre 1931, citée par Thomas Augais, Trait pour trait. Alberto Giacometti et les écrivains par voltes et faces d'ateliers, thèse de doctorat en lettres et arts sous la direction de Jean-Yves Debreuille, Lyon, Université Lyon 2 , 2009 , p. 8. 
/50 « Misère de la poésie: "L'affaire Aragon" devant l'opinion publique", reproduit dans José Pierre (sld), Tracts surréalistes et déclarations collectives, I, 1922-1939, Paris, Le Terrain vague, 1980, p. 217.

/51 Anonyme, "Mise au point communiquée par l'Association des Écrivains Révolutionnaires», l'Humanité, 10 mars 1932 , p. 2.

/52 Cette autocritique est publiée sous le nom de "Déclaration au Secrétariat de l'Union Internationale des Écrivains Révolutionnaires ", Littérature de la Révolution mondiale $\mathrm{n}^{\circ} 2$, février 1932, p. 62. Elle est reproduite en mars 1932 dans la brochure « Paillasse! (fin de "L'Affaire Aragon") ", publiée par les surréalistes.

/53 « Lettre autocritique d'Aragon et de Sadoul », José Pierre (sld), op. cit., p. 185.

/54 À propos de la confidentialité de cette déclaration et des conditions de sa publication voir Pierre Juquin, Aragon, un destin français. Le temps des rêves (1897-1939), Paris, Éditions de la Martinière, 2012, p. 520. révolutionnaire. En tant que chef de file des surréalistes, Breton répond à ses détracteurs et publie le tract «Misère de la poésie: “L'affaire Aragon" devant l'opinion publique/50 ». Dans ce texte la conception idéaliste hégélienne de l'art est mise en avant comme étant compatible avec la révolution prolétarienne. De plus, il conteste le Congrès de Kharkov qui, selon lui, tend à unifier sous le signe d'un matérialisme réducteur toute production artistique révolutionnaire. Les relations entre les surréalistes et le PCF sont alors des plus tendues lorsque l'Humanité publie le 10 mars une "Mise au point communiquée par l'Association des Écrivains Révolutionnaires » dans laquelle il est écrit: "Notre camarade Aragon nous fait savoir qu'il est absolument étranger à la parution d'une brochure intitulée: "Misère de la Poésie [...]". Il tient à signaler clairement qu'il désapprouve dans sa totalité le contenu de cette brochure et le bruit qu'elle peut faire autour de son nom, tout communiste devant condamner comme incompatibles avec la lutte de classes, et par conséquent comme objectivement contre-révolutionnaires, les attaques que contient cette brochure/51. ».

Pour la première fois, l'Association des Écrivains Révolutionnaires publie un communiqué et c'est pour annoncer qu'elle se désolidarise des surréalistes. Ce qui est étonnant, c'est la place d'Aragon dans ce communiqué. Ce dernier a probablement subi des pressions de la part du Parti, comme nous avons pu l'observer dans la lettre de Fréville à Jasienski. Sans doute a-t-il eu connaissance de la publication de l'autocritique qu'il a dû rédiger avec Sadoul lorsqu'ils étaient à Kharkov/52. On peut y lire que les deux congressistes de Kharkov y ont non seulement renié le "Second manifeste du surréalisme », « dans la mesure où il contrarie le matérialisme dialectique » et que dorénavant ils repoussent "toute idéologie idéaliste (notamment le freudisme) "; mais qu'ils ont également reconnu « avoir eu tort en attaquant hors des organes du Parti deux membres du PCF (les camarades Barbusse et Caby)/53 ".

Publier cette déclaration plus d'un an et demi après le Congrès et en pleine "Affaire Aragon » alors qu'elle était normalement réservée aux canaux confidentiels de l'IC/54 interroge. Il est probable qu'elle mette Aragon face à ses contradictions et qu'elle divise les surréalistes afin de les évincer, en tant que groupe à part entière, de la future Association. Lorsque les surréalistes ont connaissance de cette déclaration (probablement à la mi-mars), le groupe se scinde : Aragon et Sadoul quittent Breton et entraînent avec eux Maxime Alexandre et Pierre Unik dans les rangs du PCF.

Le 17 mars 1932, une assemblée constitutive présidée par Francis Jourdain et un présidium d'honneur composé par Léopold Averbach, 
Théodore Dreiser, Maxime Gorki, André Marty, Romain Rolland et Edmond Fritsch/55 officialise la création de l'AÉAR. Une «Commission exécutive ", dont fait partie Aragon, a pour charge de désigner le Bureau de l'Association. Celui-ci est alors essentiellement composé de membres du Parti: Vaillant-Couturier est nommé Secrétaire général, Fréville occupe le poste de secrétaire adjoint et Moussinac est l'un des trois trésoriers. Le " Manifeste de l'AÉAR » publié quelques jours plus tard donne des précisions sur les intentions de l'Association. Elles sont semblables aux "principes généraux » édictés par l'AAÉR quelques mois auparavant en ce qui concerne l'organisation de l'art révolutionnaire et sa relation avec le prolétariat. De la même façon, les publications «contre-révolutionnaires » et notamment Monde de Barbusse sont condamnées. Enfin, à la différence de l'AAÉR, l'auteur du «manifeste » fustige «la base idéaliste du surréalisme [et] fait confiance à certains surréalistes pour abandonner leurs conceptions, et se rallier au matérialisme dialectique/56 ». À cette date, le PCF remporte une victoire, Barbusse n'est plus garant de l'art révolutionnaire et si les surréalistes sont finalement admis dans l'Association avec réticences et conditions, leurs voix importent peu. En 1935, les relations entre les surréalistes, l'AÉAR et le PCF, sont définitivement rompues/57.

\section{Conclusion}

Plusieurs raisons peuvent expliquer l'échec des surréalistes à constituer une organisation en lien avec l'Internationale communiste. Premièrement, leur conception "idéaliste » qui leur fait envisager l'art comme une explication et une interprétation du monde à travers la sensibilité (imagination et rêve) de l'artiste s'oppose, de fait, aux thèses matérialistes des communistes. Deuxièmement, les surréalistes refusent toute soumission à ce champ, tout en dénonçant le principe de «l'art pour l'art». Cette ambivalence fait que leur préoccupation politique et idéologique est finalement restreinte par leur activité artistique. En résulte un enfermement qui leur fait oublier des questions fondamentales comme, par exemple, l'économie. Pour Jacques Beauffet, cette situation « les accule bientôt à distinguer une révolution qui serait de l'ordre des idées, d'une révolution sociale qui poserait, elle, le problème économique/58 ». Incapables de se saisir de cette dimension économique (et donc sociale), les surréalistes se réfugient dans le champ exclusif de la culture et font finalement une partition entre l'art et le réel, qu'ils proposent pourtant d'abolir.

Enfin, cet échec doit s'étudier au prisme des rapports qu'entretiennent les deux entités hétérogènes que sont les surréalistes et le Parti communiste. Il y a d'un côté un groupe restreint composé
/55 Respectivement: soviétique, membre dirigeant de l'UIÉR; écrivain et compagnon de route américain; écrivain soviétique; dirigeant du PCF, héros des mutineries de la mer Noire; écrivain proche du PCF; chômeur tué par la police au cours d'une manifestation quelques heures avant la tenue de l'assemblée constitutive.

/56 Anonyme [Paul Vaillant-Couturier], "Le manifeste de l'Association des Écrivains et Artistes révolutionnaires ", l'Humanité, 29 mars 1932, p. 4 .

/57 Dès 1933,

André Breton, René Crevel et Paul Éluard sont exclus du PCF pour avoir refusé de désapprouver la publication de Ferdinand Alquié dans laquelle il dénonce le « vent de crétinisation systématique, qui souffle d'URSS ». Voir Ferdinand Alquié, "Lettre », Le Surréalisme au service de la Révolution $\mathrm{n}^{\circ} 5,1933$, p. 44. En juin 1935, lors du Congrès international des écrivains pour la défense de la culture, les surréalistes dénoncent le régime stalinien. La rupture est alors consommée.

/58 Jacques Beauffet, «Surréalisme et théorie de l'art, les années trente » dans Jacques Beauffet, Madeleine Bonnard, Bernard Ceysson, et al., L'art dans les années trente en France, cat. exp. 
(Saint-Étienne, Musée d'Art et d'industrie, marsmai 1979), Saint-Étienne, Musée d'art et d'industrie, 1979, p. 61.

/59 Afin d'assoir leur hégémonie littéraire les communistes mènent des attaques contre certains groupes d'écrivains de gauche comme les populistes et leur revue Nouvel Âge dirigée par Henry Poulaille; ou encore contre les disciples d'Henri Barbusse qui collaborent à Monde. Ce journal est d'ailleurs la cible de Paul VaillantCouturier qui souhaite, en 1932, que le Parti en prenne le contrôle. À ce sujet voir Paul Vaillant-Couturier, «Monde?Non! Un Nouveau Monde? Oui! », l'Humanité, 28 juin 1932, p. 4. d'écrivains et d'artistes et de l'autre un parti politique affilié à un système international ayant accès à des ressources logistiques et financières considérables et fort déjà de plusieurs milliers de membres parmi lesquels se trouvent des écrivains et des artistes. De cette façon, si dans les années 1930-1932 il semble que l'une des principales ambitions des surréalistes soit de créer une association d'artistes et d'écrivains révolutionnaires en collaboration avec le $P C$, cette recherche d'alliance n'est pas réciproque. Du point de vue des surréalistes la question de leur adhésion au PCF et de leur lien avec le Komintern est donc centrale, alors que du point de vue du Parti elle n'est que secondaire.

Cette domination n'est pas seulement symbolique, elle réside également dans les stratégies mises en place par l'organisation politique pour arriver à ses fins. L'adhésion des surréalistes au PCF et de fait leur intrusion dans le champ culturel et artistique du Parti intervient au moment où ce dernier est en désaccord avec Barbusse qui est pourtant censé en incarner la ligne. Le conflit ouvert entre l'écrivain et les surréalistes devient alors un moyen pour les communistes d'exercer une pression sur le directeur de Monde afin qu'il se conforme à leurs directives, même si c'est en vain. De la même façon, la volonté des surréalistes de jouer un rôle dans le mouvement communiste international les place, malgré eux, dans une position de dominés dont ils n'arrivent à sortir qu'au prix d'une violente rupture qui les fragilise. Cette conjoncture, créée en partie par le PCF, lui permet d'avoir le contrôle exclusif d'une organisation littéraire et artistique à l'ambition nationale et au réseau international.

Cet épisode est révélateur de la volonté du PCF qui, guidé par l'exemple de l'URSS, souhaite s'immiscer dans tous les domaines de la vie intellectuelle et culturelle. Avec l'AÉAR, le parti politique inaugure cette pratique. Toutefois, au début des années 1930, l'action des communistes français se concentre davantage sur l'instrumentalisation des groupes artistiques et littéraires (surréalistes, populistes, barbussiens, etc.) plutôt que sur la création artistique en elle-même/59. Bien sûr, le Parti, émet des souhaits quant à une forme d'uart révolutionnaire" qu'il voudrait voir émerger mais celui-ci n'est pas encore clairement défini. Il faut attendre 1934 pour que le réalisme socialiste, en tant que méthode de création fasse son apparition en France, mais il ne trouvera guère d'écho à ce moment-là. C'est à la fin des années 1940, en pleine guerre froide, alors que le parti communiste cherche à maintenir son hégémonie, qu'il se met à intervenir dans les questions artistiques comme jamais auparavant. En juin 1947, lors du XI Congrès National du PCF, Laurent Casanova annonce que le Parti communiste « ne peut 
adopter une attitude indifférente à l'égard de l'esthétique/60 ", il en appelle alors à l'émergence d'un art qui soit le reflet de l'action du Parti. À partir de là, il ne sera plus question d'instrumentalisation de groupes mais d'une réelle ingérence du Parti dans le champ artistique. Quant aux surréalistes, leur exclusion du PCF, puis de l'AÉAR les amènent à créer leur propre organisation artistique révolutionnaire. Celle-ci sera structurée avec l'aide de Léon Trotski et Diego Rivera lors d'un voyage de Breton au Mexique. En 1938, les trois hommes créent l'éphémère Fédération Internationale de l'Art Révolutionnaire Indépendant (FIARI) dont le manifeste intitulé "Pour un art révolutionnaire indépendant » constitue une violente attaque contre le régime stalinien.
/60 Laurent Casanova, "Le Communisme, la pensée et l'art », discours prononcé au $11^{\mathrm{e}}$ Congrès national du Parti Communiste Français, Strasbourg, 25-28 juin 1947, Paris, Éditions du Parti Communiste Français, 1947, p. 13.

\section{Gwenn Riou}

